\title{
Bio Metric TB Notification System Interfaced with IOT
}

\author{
${ }^{1}$ Mrs. S.Kanagamalliga, Asst. Professor I/ECE Dept, \\ malliga87@gmail.com \\ ${ }^{2}$ M.Naveen Chandran ECE Dept, \\ Velammal College of Engineering and Technology, Viraganoor, Madurai \\ navenrascal@gmail.com
}

\begin{abstract}
In Medical fields we are monitoring various parameters (Blood Pressure, ECE, CO2, etc...) of the patient using internet of things. The real-time parameters is used to monitor patient's health and sent threw Internet using cloud technology. These parameters are sent to a remote Internet location so that user can view these details from anywhere in the world. The aim of the project is to measure the value of Micro Bacterium Tuberculosis (MTB) patients with help of biosensor. The Biosensor is used to detect the coughing level and it indicate the range of TB. The heat sink in Biosensor is used to detect Air Pressure when person is coughing. Depending on pressure level, the ranges are calculated. If the pressure level is medium, it will give warning to patients by alarm,so they can take vaccination and medicine. If the pressure level is high, the signal and location will be sent to the specific number of patients. By utilizing GPS, the location of patient it will be sent to respective hospital and specific number by indicating that the patient is in series condition, and patient history will also sent to hospital. This is used to reduce the death level of TB patients.
\end{abstract}

Keyword : IOT, Biometri, TB

\section{INTRODUCTION}

The intention of this research is to establish a relationship between dairy cattle diseases with various non-invasive sensors for the development of a health monitoring system[1]. It also explains how the primary sensors identified by this research can be used to forecast cattle health in a simple, basic manner. The scope of this paper is limited to the discussion about the non-invasive, wearable sensors that are needed to determine the cattle diseases. Biomedical (BM) is the application of engineering principles and design concepts to medicine and biology for healthcare purposes (e.g. diagnostic or therapeutic). Unprecedented precision in the diagnostic. Continuous health monitoring can provide long-term information about the evolution of physiological indicators (e.g. blood pressure, pulse, insulin level, EKG, etc.).
At the same time, minimally invasive diagnosis is getting more precise (e.g. oncology, etc.)[2].

Tuberculosis (TB) is an infectious disease usually caused by the Mycobacterium tuberculosis (MTB)[3].Tuberculosis generally affects the lungs, but can also affect other parts of the body. Adherence to tuberculosis (TB) treatment is important for promoting individual and public health. Poor adherence results in more individual suffering and death as well as more costly treatment as treatment regimens lengthen and drug resistance develops[4].

\section{DETAILS EXPERIMENTAL}

\subsection{Materials and Procedures}

A biosensor is an explanatory gadget, utilized for the identification of an investigative that joins an organic segment with a physicochemical indicator. The touchy organic component, e.g. tissue, microorganisms, organelles, cell receptors, 
chemicals, antibodies, nucleic acids, and so on., is an organically inferred material or biomimetic part that communicates, ties, or perceives with the expository under study[5]. In this task, we are observing different parameters of the patient utilizing web of things. In the patient observing framework in light of Internet of things venture, the continuous parameters of patient's wellbeing are sent to cloud utilizing Internet network. These parameters are sent to a remote Internet area with the goal that client can see these points of interest from anyplace on the planet.

There is a noteworthy contrast between SMS based patient wellbeing checking and IOT based patient observing framework. In IOT based framework, subtle elements of the patient wellbeing can be seen by numerous clients. The explanation for this is the information should be observed by going by a site or URL. Though, in GSM based patient checking, the wellbeing parameters are sent utilizing GSM by means of SMS[6].

Adherence to tuberculosis (TB) treatment is imperative for advancing individual and general wellbeing. Poor adherence brings about more individual enduring and passing and additionally more exorbitant treatment as treatment regimens protract and sedate obstruction develops. The significant target of this framework is to make foundation of TB investigation more intelligent and to actualize a noninvasive wearable to track physiological and natural exercises of steers utilizing Internet of Things false name IoT[7].

\subsection{WORKING PRINCIPLE}

Integrated image collecting and algorithm chip together with finger print scanner. By using finger print scanner the TB patients details have been save in Arduino board. The Bio-sensor is used to detected the coughing and finally is indicate the range of TB. The principle behind bio-sensor using heat sink which detects the Air pressure when person is coughing. By using the pressure force \& frequency the value has send to the ardino board. Our kit and Bluetooth device have been paired. The ardino send the value to the mobile app (TB Monitor) by using Bluetooth device.

Depending on pressure level the ranges are calculated. If the level is medium it will give warning to patients. If the level is high the signal and location will be send to the specify number for patients. so by utilizing GPS the location of patient will be send to respective hospital by indicating that the patient is in series condition, and patient history will also send to hospital. TB investigation equipment unit is labeled with a wearable gadget. The wearable gadget and sink hub is outlined in light of design of gadget to GSM network. All information alongside time and date can be extricated as an exceed expectations sheet for promote investigation. Therefore general wellbeing and body temperature will be enhanced by diminishing human wellbeing examination costs guaranteeing little size, minimal effort, high consistency and dependability.

The ardino will also show the patients Id while they place the finger in finger print scanner and coughing the value and enroll Id will also show in lcd display. The application of the LCD display is $2 \times 16$ alphanumeric display and has Two rows each with 16 column spaces , 8 pins for data , 3 pins for control (RS,RW and EN), Contrast and Brightness Adjustable. It can also adjust the Backlight ON/OFF functionality for energy conservation.

\section{RESULTS AND DISCUSSION}

\subsection{The Working flow of MATLAB Platform}

For our innovation to take the TB coughing values the MATLAB has used. By using MATLAB and Bio-Sensor we have test for 5 TB patients to analyses. The wave of TB 
coughing when for 1 patient cough in BioSensor. The Arduino will test via MATLAB program by the given specific level range and analyses the graph. They method have repeated for 5 patients and output of graph have taken at last. By using 5 graph we have analysis the range for high level patients. Then we have apply the output and save in ardino. If the level of graph range value have high for upcoming patients then it identified that it is high range the (series condition) of patient. The in app the specific number of patient by knowing Enroll id value of TB and location to them.

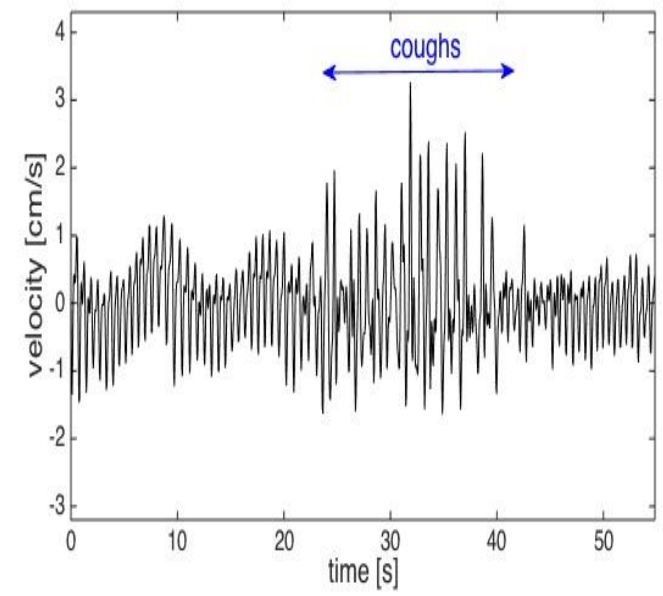

Figure 1: waveform of patient coughing level

\begin{tabular}{|c|c|c|c|}
\hline Patient- & & Patient- & \\
\hline TB Monitor & ! & TB Monitor & $\vdots$ \\
\hline Devi & is Connected & & is Connected \\
\hline ID Matched & 67 & ID Matched & 63 \\
\hline Analog Value & 25 & Analog Value & 35 \\
\hline Enroll ID & 67) & Enroll ID & 63 \\
\hline 9442495817 & Pick Mobile & 9442495817 & Pick Mobile \\
\hline Enroll & Send SMS & Enroll & Send SMS \\
\hline
\end{tabular}

\section{Figure 2: Output value of TB level Monitoring in app}

TB examination equipment unit is labeled with a wearable gadget. The wearable gadget and sink hub is planned in view of engineering of gadget to GSM arrange. All information alongside time and date can be extricated as an exceed expectations sheet for advance examination. Accordingly general wellbeing and body temperature will be enhanced by diminishing human wellbeing review costs guaranteeing little size, minimal effort, high consistency and unwavering quality.

\section{Advantage}

- 'Wearable' gadgets are smaller than normal electronic gadgets worn on the body, frequently incorporated with or intended to supplant existing extras, for example, a conservative gadget.

- This advertise section is blasting, empowered by Internet of Things innovation. In this manner the requirement for littler, more instinctive gadgets is quickly expanding.

\section{CONCLUSION}

The venture "BIO METRIC TB NOTIFICATION SYSTEM INTERFACED WITH IOT" has been finished effectively and the yield comes about are confirmed. The outcomes are in accordance with the normal yield. The venture has been checked with both programming and equipment testing apparatuses. In this work "LCD, PIC Microcontroller, Resistive Sensor and Capacitive sensor" are picked are turned out to be more proper for the proposed application. The undertaking is having enough roads for future upgrade. The undertaking is a model that satisfies all the 
coherent necessities. The undertaking with negligible enhancements can be specifically appropriate for ongoing applications. Accordingly the venture contributes a noteworthy advance forward in the field of "Cutting edge AUTOMATION", and further clears a street way towards quicker improvement $\mathrm{s}$ in a similar field. The undertaking is further versatile towards constant execution and fringe up degrees. This work can be connected to assortment of modern and business applications.

\section{REFERENCE}

1. World Health Organization (WHO), Global Tuberculosis Report, 2015 (Available at: http://www.who.int/ tb/publications/global report/en/.).

2. World Health Organization (WHO, Geneva), Systematic Screening for Active Tuberculosis: Principles and Recommendations, 2013 (Available at: $\quad$ https://www.ncbi.nlm.nih.gov /books/NBK294083/).

3. M.D. Perkins, J. Cunningham, Facing the crisis: improving the diagnosis of tuberculosis in the HIV era, J. Infect. Dis. 196 (2007) S15-27.

4. S.D. Lawn, M.P. Nicol, Xpert@MTB/RIF assay: development, evaluation and implementation of a new rapid molecular diagnostic for tuberculosis andrifampicin resistance, Future Microbiol. 6 (2011) 10671082.

5. M. Duman, E. Piskin, Detection of Mycobacterium tuberculosis complex and Mycobacteriumgordonae on the same portable surface Plasmon resonance sensor, Biosens. Bioelectron. 26 (2010) 908-912.

6. R.K. Daher, G. Stewart, M. Boissinot, M.G. Bergeron, Recombinase polymerase amplification for diagnostic applications, Clink. Chem. 62 (2016) 947-958.

7. V.S.Roshni, K.Brinda , S.Ranjani, "BEACON: AN APPLICATION OF IOT", International Journal of Innovative Research in Applied Sciences and Engineering, Volume 1, Issue 12, 2018 pp.241-244. 